\title{
Accessing e-Learning Systems via Screen Reader: An Example
}

\author{
Maria Claudia Buzzi ${ }^{1}$, Marina Buzzi ${ }^{1}$, and Barbara Leporini ${ }^{2}$ \\ ${ }^{1}$ CNR-IIT, via Moruzzi 1, 56124 Pisa, Italy \\ Claudia.Buzzi@itt.cnr.it, Marina.Buzzi@itt.cnr.it \\ ${ }^{2}$ CNR-ISTI, via Moruzzi 1, 56124, Pisa, Italy \\ Barbara.Leporini@isti.cnr.it
}

\begin{abstract}
The evolution of the Information and Communication Technology (ICT) and the rapid growth of the Internet have impelled the pervasive diffusion of e-Learning systems. This is a great opportunity for visually-disabled people provided that both the interactive environment, created by the Learning Management Systems, and the Learning Objects, created by teachers, are properly designed and delivered. In this paper we investigate interaction of the blind user with an open source Virtual Learning Environment (Moodle) and discuss how the use of the W3C Accessible Rich Internet Applications (ARIA) suite may improve the experience of navigation via screen reader.
\end{abstract}

Keywords: e-Learning, accessibility, usability, blind, ARIA.

\section{Introduction}

Today Learning Management Systems (LMSs) offer many educational tools from a single environment: web courses, exercises, chats, wikis, self-assessment SW, surveys, forums, podcasts and even more. User Interfaces (UIs) then become richer with embedded videos, text, sounds, customized widgets. Accessibility and usability of eLearning systems and objects are crucial for providing an easy and satisfying experience to all. Specifically, Virtual Learning Environments (VLEs) should be friendly and simple to use for everyone, in order to eliminate any "technical barriers" to the learning process and allow students to concentrate on content.

However, despite considerable research focus in this field, interacting with a virtual environment and using learning objects is still difficult for a blind user who cannot see the screen and is unable to use a mouse. Furthermore, interaction requires the aid of assistive technology (i.e., screen reader and voice synthesizer), which adds another degree of complexity.

Designers of e-Learning systems must consider three crucial factors: usability, accessibility, and educational effectiveness. Consequently, the challenge is to design systems that are simple to use and accessible to all, while maintaining pedagogical and educational efficacy. In particular, blind students may fruitfully utilize e-Learning systems if educational materials are accessible and learning paths can be tuned to the "rhythm" of the individual student. 
When designing for blind users, it is necessary to consider the three main interacting subsystems of the Human Processor Model: the perceptual, motor and cognitive systems [5]. Sightless persons perceive page content aurally and navigate via keyboard. This makes the "reading process" time-consuming and sometimes difficult and frustrating, if the contents are not designed with special attention to their needs. The cognition part of the interaction is important, since many learning techniques are only relevant to people with good vision and may not apply to someone with a visual impairment. Thus, alternative ways to deliver the same content should be provided. Furthermore, a blind person may develop a different mental model of both the interaction and the learning processes, so it is crucial to provide an easy overview of the system and contents. Non-visual perception can lead to:

1. Content serialization. The screen reader reads the contents sequentially, as they appear in the HTML code. This process is time-consuming and annoying when part of the interface (such as menu and navigation bar) is repeated in every page. As a consequence, blind users often have to stop the screen reading at the beginning, and they prefer to navigate by Tab Keys, from link to link, or explore the content row by row, via arrow keys.

2. Content and structure mixing. The screen reader announces the most important interface elements such as links, images, and window objects as they appear in the code. For the blind user, these elements are important for figuring out the page structure, but require additional cognitive effort.

3. Table. If the table's content is organized by columns the screen reader (which reads by rows) announces the content of the page out-of-order, and consequently the information might be confusing or misleading for the user.

4. Lack of context. When navigating by screen reader the user can access only small portions of text and may lose the overall context of the page; thus it may be necessary to reiterate the reading process.

5. Lack of interface overview. Blind persons do not perceive the overall structure of the interface, so they can navigate for a long time without finding the most relevant contents.

6. Difficulty understanding UI elements. Links, content, and button labels should be context-independent and self-explanatory.

7. Difficulty of working with form control elements. The new JAWS version (v. 10) simplifies the interaction with forms since it can automatically activate the editing modality (for text input) when the virtual focus arrives at the text box (for instance when the user presses the tab key). However, with previous screen reader versions the user may have great difficulties since switching between exploration and editing modalities is required (i.e. form mode on/off).

8. A blind person is unable to access multimedia content such as video streaming, video conferencing, and captioning. If an alternative description is not present the user may lose content.

In this paper we analyze accessibility and usability for the blind of two demo courses offered by Moodle (http://www.moodle.org/), a very popular open source eLMS. Then we illustrate how ARIA, the suite developed by the Web Accessibility Initiative (WAI) group of W3C, may facilitate interaction for the blind. Lastly, the paper presents our conclusions. 


\section{Related Works}

E-learning systems pose new challenges with respect to classic user-centered product design, where the target is a set of homogeneous users. Learner-centered Design must answer to the needs of multiple learner categories due to differences in learning strategies, know-how, experiences, motivation to learn and, not least, user age and ability. If appropriately designed and implemented, e-Learning systems are more effective and useful than classroom learning [4]. Various studies focus on the usability of e-Learning systems and some also include a general discussion on accessibility, but to our knowledge only a few focus on totally blind persons.

Fifteen years ago Nielsen proposed an informal method for evaluating systems and design usability based on verifying the conformance to a set of principles of usable software design (heuristics) performed by experts [8]. This approach, which detects a high percentage of problems, is cost-effective and easy to implement compared to usability testing. However, to be effectively applied to the e-Learning domain these general principles needed to be further refined. A few years later, Squires and Preece embedded usability heuristics in the socio-constructive theory and specified criteria ad hoc for e-Learning [12]. However, even today various researchers criticize the lack of accurate studies in this field. In [1] the authors take the first steps in defining a methodology for the rigorous evaluation of e-Learning applications, but accessibility for special needs students is not analyzed. Furthermore, Zaharias critically examined the usability of e-Learning applications and proposed a new usability measure: the student's intrinsic motivation to learn [15]. Developing a usability evaluation method based on a questionnaire, he carried out two large empirical studies showing the reliability of this approach. For Sloan et al. the goal of universal accessibility on the Web is inappropriate and instead it is necessary to explore multiple routes to provide equivalent experiences [11]. As Kelly et al. argued, rather than demanding that an individual learning resource be universally accessible, it is the learning outcome that needs to be accessible [7]. Based on user profiles, metadata and dynamic connection to resources, the user's experience can be customized to match his/her abilities. Then an appropriate design is crucial for improving the accessibility and usability of eLearning Systems.

De Marsico et al. [5] defined methodological guidelines involving users with disabilities as well as pedagogical experts in the development process, believing that input of different know-how may enrich the quality of e-Learning applications, and provide a more satisfying learning experience. They also include two examples of building and providing learning objects accessible respectively to visually- and hearing- impaired students. Rodriguez et al. describe a project for improving e-Learning experience for the visually impaired, based on ethnomethodology and taking into account psychosocial issues, the user context and experience [9]. Next they created different learning object formats suitable for the blind, including DAISY (Digital Accessible Information SYstem). However, although authors describe the methodology used to improve learning materials no general guidelines are offered to the reader. Within the framework of a project aimed at providing an accessible e-Learning platform for disabled and adult learners, Santos et al. [10] illustrate a methodology for developing standard based accessible courses which use two-step evaluations. However for the totally blind, more specific UI features are necessary than those provided 
in this study, such as providing a page overview, full control of interface elements and easy and rapid navigation via keyboard.

E-Learning is a great opportunity for visually-disabled people, provided that both the interactive environment (created by the LMS) and the learning objects are properly designed and delivered. In this paper we mainly focus on accessibility and usability of the virtual environment for the blind, taking as an example the environment offered by Moodle. Concerning Moodle, Debevc et al. [4] compared usability of two LMSs, i.e. Moodle vs a proprietary system, when delivering an identical package of educational materials. This usability study was carried out with a SUMI (Software Usability Measurement Inventory) evaluation that is not specific for e-Learning environments. However indications derive from this study, that point out Moodle's weaknesses (vs the proprietary system) in efficiency as well as learnability.

\section{The Moodle Virtual Learning Environment}

To evaluate accessibility and usability of a Virtual Learning Environment (VLE) via screen reader, we chose Moodle, an Open Source LMS which offers a rich VLE. Thanks to its large spectrum of available tools and options it has become very popular worldwide. Specifically it offers (http://www.moodle.org): Assignment modules, Blogs, Chat, Choices with multiple response options, Course resources (Moodle pages, uploaded files or web links), Databases, Forums, Glossaries, Interaction, Lessons, SCORM packages, Surveys, Quiz module, Wikis and Workshop modules. The richness of the VLE is Moodle's strength but the environment's complexity can also create difficulties in interaction via screen readers.

\subsection{Evaluation Methodology}

In the first step of our research, we analyzed two demo courses available on the website (http://demo.moodle.org) by using the screen reader JAWS for Windows (http://www.freedomscientific.com) v. 9.0 and 10. We used both the MS IE version 7.0 and the Mozilla Firefox version 3.0.5 browsers.

The test was carried out by all the authors independently; afterwards, outcomes were compared and integrated. One author has been totally blind since childhood and uses the JAWS screen reader every day; thus she knows this tool's functions very well and is able to use advanced commands. By analyzing the test results we noticed that in spite of her great expertise using JAWS, she was unable to perceive the exact structure of the layout. This paper's sighted authors carried out the test using only JAWS basic commands, but viewing the Moodle UI allowed them to rapidly understand the origin of obstacles encountered when interacting via screen reader. Therefore, integrating both these outcomes led to a more accurate analysis. The different experiences of the authors when using JAWS allowed us to cover a variety of interaction modalities: i.e. both basic commands, which simulate the use of beginner users, and advanced screen reader functions.

\subsection{Exploring the Moodle Demo}

To illustrate how a blind user interacts with the Moodle environment, we explore several pages related to two selected courses, according to specific features to be 
tested: main page functions, topic outline of a selected course, interacting environment, and so on.

When opening the demo page, before doing the log-in, JAWS announces four headings. Through the special command "Insert+F6" JAWS shows the headings list. Although different levels are assigned to these headings, the levels order used is unsuitable. In fact, level 1 is associated with the course title; thus, by pressing the key " 1 " the virtual focus moves to the beginning of the page. In this case, it would be more appropriate to assign the level 1 to the "Log in" section, which logically is the first part of the interface the user must navigate to enter the system.

After the login, the list of available courses is shown. Once the chosen course is clicked, its page is loaded and opened. The screen reader informs the user about headings as well as the number of links. Basically, this page seems to be accessible, because several accessibility features are detected (for instance, a hidden link pointing to the "main content") by the screen reader. Also, headings are immediately identified and announced by JAWS. But, are all these features suitable for comfortable navigation by blind users? Let us consider the "Higher Education Film Studies Module" (in the following referred to as Course A), available as a demo course.

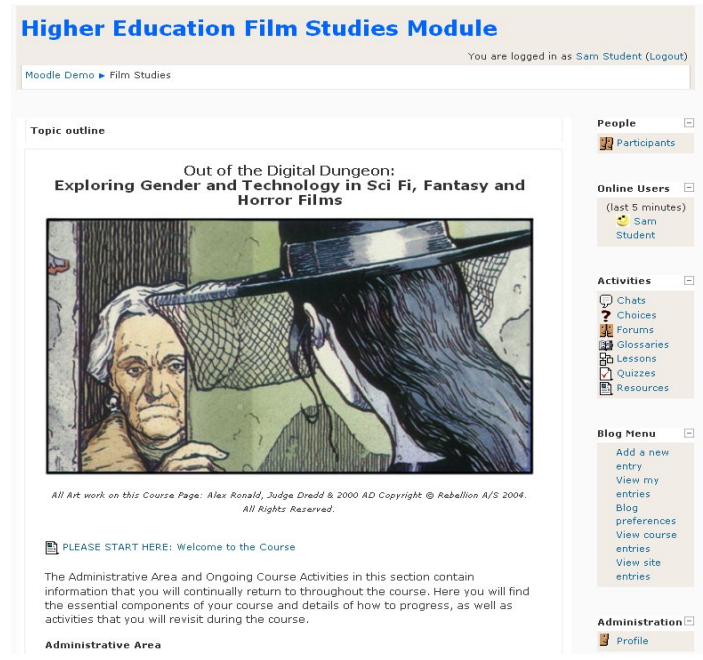

Fig. 1. Moodle demo - Course A

After selecting this course the following elements are detected by JAWS:

- Headings: 32 headings are announced. By exploring the heading list (Insert+F6 command) we note that most of them refer to the course, but the others are related to the e-learning tools (e.g. chat, blog, and so on). Those headings are too numerous to be navigated comfortably. Although headings are used, the page is too long to be read by keyboard via screen reader. Furthermore, it is not very clear which headings refer to the course modules and which ones to the interacting platform functions (see below). 
- Layout table: to show the Topic outline a table has been used for the layout. Specifically two tables are used, the second nested in the first cell of the first table, but JAWS announces only one table. Although headings are used to split the long content, a table should not be used for the rendering. Moreover, the "summary" attribute of the table is "table layout", totally useless. A more appropriate summary value could be "table of contents"; in this way, by just pressing the letter " $t$ " JAWS will announce immediately "Table of contents". If a layout table is used, at least a meaningful summary should be applied.

- Number of links. The page contains too many links (in our case 115 links).

- Images. Many of the images in the pages of the course are not perceived by the screen reader since no alternative text is provided or descriptions are negligible. After the log-in as teacher, we tried to insert an image and found that the alternative text is now mandatory. However there is no control on the number of characters inserted, so it is possible to skip this edit field with only a space press.

In order to understand whether the issues encountered in the selected course could be generalized, we attempted to navigate another demo course. Specifically, we picked up the course "Moodle Features Demo" (in the following referred to as Course B). Also the main page of the second course includes a great number of links, two tables used for visual layout, and 25 headings. However, the level assigned to each heading as well as their arrangement differ from Course A. In this case, first the headings for interacting with the Moodle environment are available, and then the Topic outline. Consequently, it seems the UI is not consistent among the courses even if they are very similar (probably due to changes in the Moodle versions used to create these courses).

However, headings arranged in this way might make the page structure somewhat unclear. In fact, it is difficult to know how many modules compose the course and their titles and contents, since the module number has not been included in the $<\mathrm{Hn}>$ tag and thus does not appear in the headings lists (Fig. 3); to obtain this information the user must read the page in a sequential way, as shown in the Fig. 2 - left section. All headings are mixed and not well grouped by clearly indicating which are modules (See Fig. 3), and which are associated with the virtual environment (on the right side in Fig. 1). In other words, the main regions: "menu", "topic outline" and "interacting actions" should be better indicated.

For example, by opening the page related to "eXe SCORM package", the screen reader recognizes some unclear elements:

- various graphical icons; the image tags have the ALT attribute, but the alternative descriptions used are not clear for the type of the icon. For instance, several links are preceded by an icon labelled "Completed" (See Fig. 2 - right section). It is not clear what this means.

- Frame: in the page a frame called "scoframe1 frame" is identified by JAWS, which is not particularly clear. Even if frame content is explored in a sequential way, is unclear what the student. According to the last version of accessibility guidelines [WCAG 2.0] frames should be avoided (deprecated).

The following figure reports the page content as it is interpreted by the screen reader JAWS. 


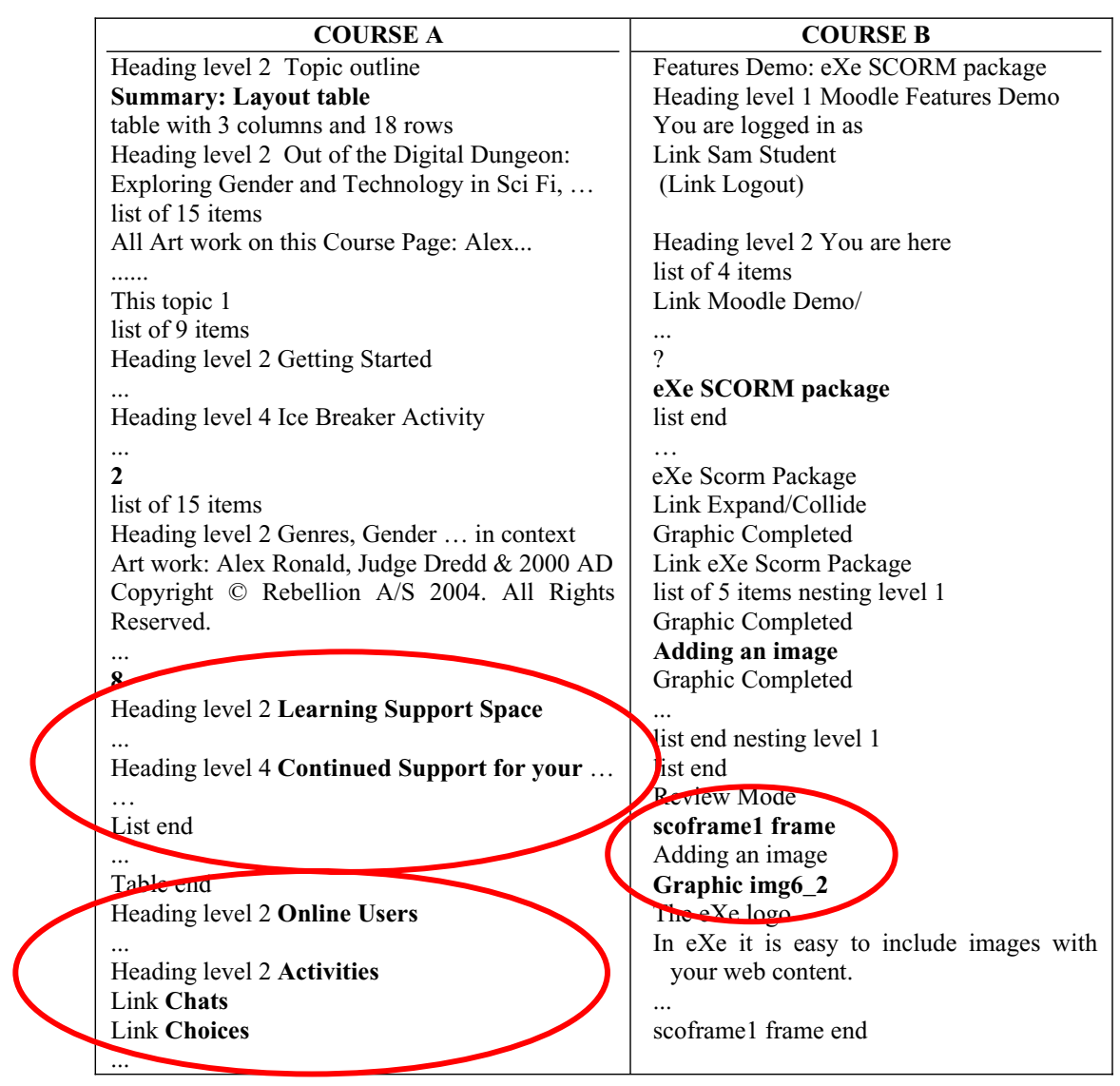

Fig. 2. Page segments sequentially read by JAWS (up/down arrows): on the left Course A main page, on the right a SCORM module function of Course B

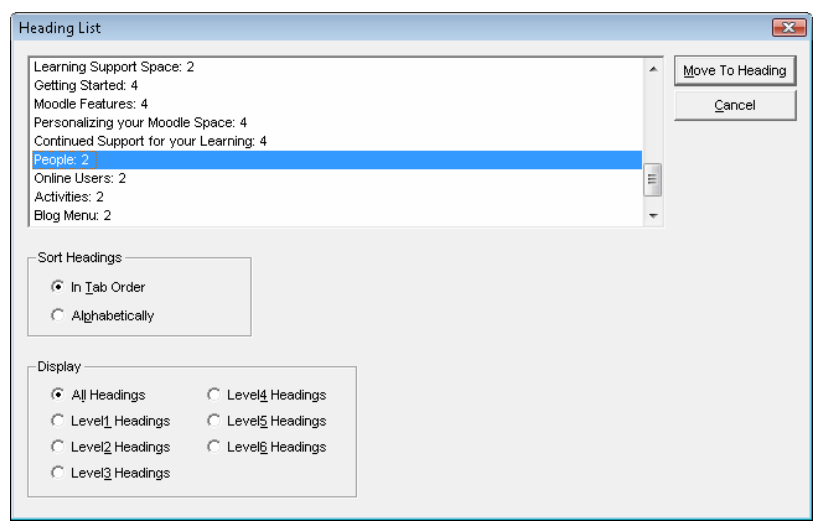

Fig. 3. List of headings of the demo course A ("Insert+F6" JAWS command) 
In short, although the pages seem to be accessible, our initial interaction using the screen reader JAWS shows some usability issues when interacting via keyboard and in a sequential manner. The few pages considered in our preliminary evaluation highlighted some difficulties orienting oneself among different information (i.e. headings and links), as well as in handling conceptual information. For instance, in some cases pictures or graphical representations are used to provide information on modules or on intersecting concepts. In a learning system it is also important to make those concepts accessible and usable by alternative modalities.

\section{Applying ARIA}

When designing a web interface it is essential to keep in mind both usability and accessibility principles. Accessibility is a basic pre-requisite for allowing access to page content, while usability guarantees an easy, simple, efficient, rapid and satisfactory navigation and interaction. Navigation is vital for special-needs persons, and in particular for the blind, because it is crucial for them to be aware of their current location on the webpage and how to return to the beginning, or how to reach a certain point in the materials [4]. The use of ARIA would enhance Moodle's usability in many ways, as explained and exhaustively illustrated by the ARIA best practises document [13]:

- Reduce the amount of unnecessary text announced. Specifically the use of Table as layout may be silently ignored by the screen reader if the table is tagged with the presentation role :

$<$ table role="presentation" $>\ldots</$ table $>$

- Definition of regions to allow the user get a page overview. Moodle utilizes heading levels to structure the page and to allow the user to perceive the whole structure. Using only standard (X)HTML code, the usability of keyboard navigation is reduced so blind users are forced to use tabbing for accessing active elements (form elements and links). To simplify interaction and allow easy jumping to main interface regions, developer usually relay on creating a link to the main content or use heading levels to structure the page (since the screen reader gives a table of headings). However the use of headings to mark sections is not consistent across web sites [13]. ARIA allows marking sections with standard specifying XHTML landmarks or defining customized regions:

$<$ div role="main" title=" Higher Education Film Studies Module "> $\ldots$

$<$ div role="region" title="Activities" $>$

- Simplifying keyboard navigation. The use of landmarks/regions also allows to simplifying navigation via keyboard since the user may jump from one region to the next by pressing a key (in JAWS v.10 the ";").

Furthermore, the developer using the attribute "flowto" defines the order in which regions should be visited:

$<$ div role="region" title="Exercises" flowto="Assessment" >

These are basic considerations to improve the interaction via screen reader with the VLE offered by the LMS, that should be integrated on the basis of all observations reported below. Further improvements are possible in the specific learning object 
prepared by the teacher; thus it is very important to provide automatic tools to support the easy creation of accessible and usable learning objects.

\section{Conclusion}

In this paper we analyze the usability of the Moodle Learning Environment for the blind. Specifically we analyzed two demo courses provided as examples by the system, highlighting features that could be improved. We mainly focus on the navigability of the virtual environment provided by Moodle, which by offering several educational tools integrated into one system, may create complex interfaces.

It is important to notice that LMSs may greatly favours the student learning process since the same educational material may be transmitted anywhere, anytime, at any learning rhythm, in a format suited to each individual's ability. On the other hand, since LMSs automatically add a virtual environment to the educational material, if the virtual environment layout is not appropriately designed with a thorough knowledge of accessibility and usability issues, it may induce problems that could be spread to the learning objects themselves. This highlights the importance of considering usability issues from the beginning of the development of every LMS.

Making a VLE suitable for the abilities and skills of all users offers many challenges. When defining the graphical UI it is fundamental to consider the needs of sighted users but the needs of the blind should also be kept in mind when writing the UI code. Specifically, the same information should be provided through both visual and auditory channels, the design should be optimized for reading via screen reader, the UIs should be easy to use via keyboard and no additional cognitive effort should be required of the blind user.

In conclusion, we believe that our findings could have general applications and that applying ARIA would enhance usability via screen reader in any Virtual Learning Environment.

\section{References}

1. Ardito, C., Costabile, M., De Marsico, M., Lanzilotti, R., Levialdi, S., Roselli, T., Rossano, V.: An Approach to Usability Evaluation of e-Learning Applications. Universal Access In the Information Society 4(3), 270-283 (2005)

2. Card, S.K., Moran, A., Newell, T.P.: The Psychology of Human-Computer Interaction. Lawrence Erlbaum Associates Inc., New Jersey (1983)

3. Debevc, M., Bele, J.L.: Usability testing of e-learning content as used in two learning management systems. European Journal of Open, Distance and E-Learning (2008), http://www.eurodl.org/materials/contrib/2008/Debevc_Bele.htm

4. Debevc, M., Verlic, M., Kosec, P., Stjepanovic, Z.: How Can HCI Factors Improve Accessibility of m-Learning for Persons with Special Needs? In: Stephanidis, C. (ed.) HCI 2007. LNCS, vol. 4556, pp. 539-548. Springer, Heidelberg (2007)

5. De Marsico, M., Kimani, S., Mirabella, V., Norman, K.L., Catarci, T.: A proposal toward the development of accessible e-Learning content by human involvement. UAIS Journal 5(2), 150-169 (2006) 
6. Eijl, P., Pilot, A., Voogd, P.: Effects of Collaborative and Individual Learning in a Blended Learning Environment. Education and Information Technologies 10(1-2), 51-65 (2005)

7. Kelly, B., Phipps, L., Howell, C.: Implementing a holistic approach to e-Learning accessibility (Retrieved), http://www.ukoln.ac.uk/web-focus/papers/alt-c2005 /accessibility-elearning-paper. doc

8. Nielsen, J.: Usability inspection methods. In: Heuristic evaluation, pp. 25-62. John Wiley \& Sons, Inc., New York (1994)

9. Rodriguez, E.P.G., Domingo, M.G., Ribera, J.P., Hill, M.A., Jardi, L.S.: Usability for All: Towards Improving the E-Learning Experience for Visually Impaired Users. LNCS, pp. 1313-1317. Springer, Heidelberg (2006)

10. Santos, O.C.B., del Viso, J.G., de la Cámara, A.F., Sánchez, S.P., Gutiérrez, C.R., Restrepo, E.: HPCN-Europe 1994. LNCS, pp. 796-805. Springer, Heidelberg (2007)

11. Sloan, D., Heath, A., Hamilton, F., Kelly, B., Petrie, H., Phipp, L.: Contextual web accessibility - maximizing the benefit of accessibility guidelines. In: Proceedings of the 2006 international cross-disciplinary workshop on Web accessibility (2006)

12. Squires, D., Preece, J.: Predicting quality in educational software: Evaluating for learning, usability and the synergy between them. Interacting with Computers 11(5), 467-483 (1999)

13. W3C. WAI-ARIA Best Practices. W3C Working Draft 4 February (2008), http://www.w3.org/TR/wai-aria-practices/

14. Wilson, R., Landoni, M., Gibb, F.: A user-centered approach to e-book design. The Electronic Library 20(4) (2002)

15. Zaharias, P.: A usability evaluation method for e-learning: focus on motivation to learn. In: Proceedings of CHI 2006 extended abstracts on Human factors in computing systems (2006) 